\title{
Oncogenic Role of Grb2 in Breast Cancer and Grb2 Antagonists as Therapeutic Drugs
}

\author{
Muhammad Ijaz ${ }^{1}$, Muhammad Shahbaz ${ }^{2}$, Wenjie Jiang ${ }^{1}$, Abdel H. Fathy ${ }^{3}$, Effat Un Nesa ${ }^{4}$, Di Wang ${ }^{1}$ and Fengshan \\ Wang*1 \\ ${ }^{1}$ School of Pharmaceutical Sciences, Shandong University, China
}

${ }^{2}$ Department of Hepatobiliary Surgery, Qilu Hospital, Shandong University, China

${ }^{3}$ School of medicine, Tongji University, China

${ }^{4}$ Department of Radiation Oncology, Qilu Hospital of Shandong University, China

Submission: March 06, 2017; Published: March 08, 2017

"Correspondence Address: Fengshan Wang, School of Pharmaceutical Sciences, Shandong University, No. 44 Wenhuaxi Road, Jinan 250012, P.R.China, Tel: +86 0531 88382589; Fax: +86 53188382548. Email addresses: fswang@sdu.edu.cn; wangfengshansdu@gmail.com

\begin{abstract}
Several adaptor proteins play dynamic roles in a number of basic cellular activities and are interconnected through different signal transduction pathways. Growth factor receptor-bound protein 2 (Grb2) is a similar adaptor molecule which was originally discovered for its major role in cell proliferation, cell survival, angiogenesis and cell differentiation. A wide range of documentation have proved the broad involvement of Grb2 in progression and development of multiple systemic malignancies including breast cancer, chronic myelogenous leukemia, hepatocellular carcinoma, human bladder cancer, etc. The mature Grb2 is 217 amino acid sequence signaling adaptor which acts as an intermediate switch between cell-surface activated receptors and downstream targets. This review is organized around three aspects, namely Grb2 protein introduction, Grb2 protein involvement in breast cancer, and the Grb2 antagonists as potential therapeutic candidates. First, we introduced Grb2 and compiled the maximum tumor conformations induced by Grb2 involvement. Numerous oncogenic pathways of Grb2 involvement and particular approaches which are potentially useful to downregulate Grb2 overexpression in breast cancer were then introduced. The activity of different types of novel peptides for the Grb2 protein was also discussed in detail. Finally, we summed up the blockade of Grb2-mediated signaling pathways directing the breast cancer, by targeting SH2/SH3 binding sites.
\end{abstract}

Keywords: Growth Factor Receptor-Bound Protein 2 (Grb2); SH2 and SH3 Domains; MAPK; Breast Cancer; Antagonist

\section{Introduction}

Several adaptor proteins play dynamic roles in a number of basic cellular activities and are interconnected through different signal transduction pathways [1]. Growth factor receptorbound protein 2 (Grb2) is a similar adaptor molecule which was originally discovered for its major role in cell proliferation, cell survival, angiogenesis and cell differentiation [2]. Recent reports have proved the Grb2 as a crucial member of immune system in the T cell development and TH cell differentiation as well [3]. On the other hand, a wide range of documentation have proved the broad involvement of Grb2 in progression and development of multiple systemic malignancies including breast cancer, chronic myelogenous leukemia, hepatocellular carcinoma, human bladder cancer, lung cancer, gastric cancer, colorectal cancer etc. [4-8]. Positive and negative regulatory roles of the Grb2 in receptor tyrosine kinase signaling makes it a double-edged sword [9]. The Grb2 is $25 \mathrm{kDa}$ adaptor protein which consists of one Src homology 2 (SH2) domain surrounded by two Src homology 3 (SH3) domains. The mature Grb2 is 217 amino acid sequence signaling adaptor which acts as an intermediate switch between cell-surface activated receptors and downstream targets (Figure 1) [10]. Grb2 through its 100 amino acid SH2 domain can recognize phosphopeptide motif pYXNX and bind directly with the sites possess the same motif like receptor tyrosine kinase (RTK); (e.g epidermal growth factor receptor (EGFR), platelet derived growth factor receptor (PDGFR), hepatocyte growth factor receptor (HGFR), etc.), non-receptor tyrosine kinases such as focal adhesion kinase (FAK), and insulin receptor substrate 1 (IRS1), and phosphotyrosine phosphatases such as SHP-2. The Grb2-SH3, a sequence of 50 amino acids hold two pockets recognized as carboxy-terminal (C-SH3) and amino-terminal (N-SH3) domains, and have ability to bind proline-rich motif [11]. The SH3 domains allow Grb2 to link with downstream effector son of seven-less (SOS) which is known as guanine nucleotide exchange factor and is a key activator of Ras. 
The Ras activation by SOS is crucial for various functions such as cell growth, B and T cell development and differentiation of stem cells [12]. Ras are unique small GTPases which take part in more than 20 signaling pathways. Ras usually exists in two states in the cytoplasm including ground state (Ras-GDP) and active state (Ras-GTP). Generally, two sets of catalytic proteins, guanine exchange factors (GEFs) and GTPase activating proteins (GAPs) are known to function as binary switches for Ras. GEFs promote the Ras from inactive state (Ras-GDP) to active state (Ras-GTP) whereas, GAPs act entirely opposite to GEFs and convert Ras from active state to inactive state. Ras on activation triggers the Raf (a serine/threonine kinase), extracellular regulated kinase (ERK) and mitogen activated protein kinas (MAPK) cascade $[13,14]$.

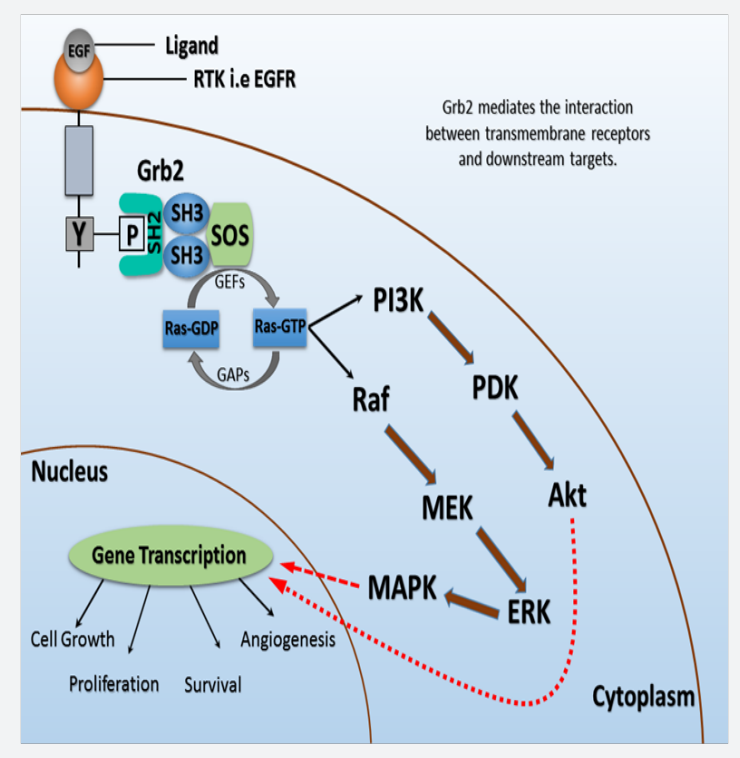

Figure 1: Schematic representation of Grb2 signaling pathway.

The cell surface receptor activation permits Grb2 to bind phosphorylated tyrosine (pTyr) kinase intracellularly. After the stimulation, Grb2 through SH3 domains activate downstream substrate SOS (a nucleotide exchange factor) which plays a crucial role in the Ras activation. The main role of SOS is to interlink Grb2 with the downstream Ras. The Ras exists in GDP-bound and GTP-bound forms, where it present in inactive and active states respectively. RasGTP complex results in the activation of Raf/ERK and finally MAPK of the Grb2 signaling pathway.

Grb2 stable expression is critical for the normal cell functions which it does through strict controls over nSH3-SH2-cSH3 structure. However, Grb2 slight lapse of these tight grips might cause the abnormal activation of downstream substrates which in turn leads to the cancer progression. Ras functioning regulated as GDP/GTP cycle is crucial for the proper signal transduction as well, where GEFS and GAPs act as binary buttons. Abrupt function of any one of both buttons might arrest Ras in either permanent active or inactive state which may contribute to malignant cell growth. In mammalian cells Ras gene is expressed in three froms namely H-Ras, K-Ras and N-Ras, and mutations in 12,13 or 61 positions in one of the three Ras genes convert them to active oncogenes which can be found in multiple tumor types $[15,16]$. Another control in Grb2 signaling is monomer-dimer equilibrium which determines, the Grb2 functioning will be either normal or oncogenic. Grb2 becomes functional for MAPK signaling pathway when it is present in monomer form, while dimer is totally opposite to it which makes Grb2 inactive for this signaling cascade. Thus proper work of this equilibrium is thought to be another switch for the regular cell functioning and a key control point in MAPK pathway [17]. Although, a slight loss of Grb2 control over these key points totally changes the entire pathway to an oncogenic track and as a result of which Grb2 launches a number of defected signaling pathways. Thus, the Grb2 wide concern in multiple malignant growth types makes it a significant target for the design of anticancer treatment in clinical arena [18].

In this review we have discussed the broad involvement of Grb2 in the progression and development of human breast cancers. Moreover, we have highlighted the maximum possible ways to efficiently tackle Grb2 in the treatment of breast malignancies which might be helpful to design potent antidotes for the Grb2-mediated breast cancers.

\section{Grb2 and Cancer}

Grb2 acts as a pivotal signaling mediator in several oncogenic signaling cascades and has been implicated in a number of human systemic malignancies. The presence of human Grb2 gene at chromosome 17 (q22) is supposed to be the key factor of its central involvement in leukemias and solid tumors [18]. This review gives a brief information regarding the extensive Grb2 concern with the malignant growth of several breast cancers.

\section{Grb2 and Breast Cancer}

Breast cancer accounts for the most frequently diagnosed cancer and the leading cause of deaths from cancer among 
females worldwide, with an approximate 1.7 million new cases and 521,900 deaths in 2012 [19]. Recently, Kapoor et al. [20] has demonstrated the prominent roles of Grb2 in proliferation and progression of systemic disorders including breast cancer and mammary tumors [20]. Another study indicated the role of mammalian Grb2 as a vital adaptor in the differentiation of mouse embryo in earlier stages which further through SOS-Ras signaling acts as rate limiting for the development of mammary carcinomas induced by polyomavirus middle T antigen [21].

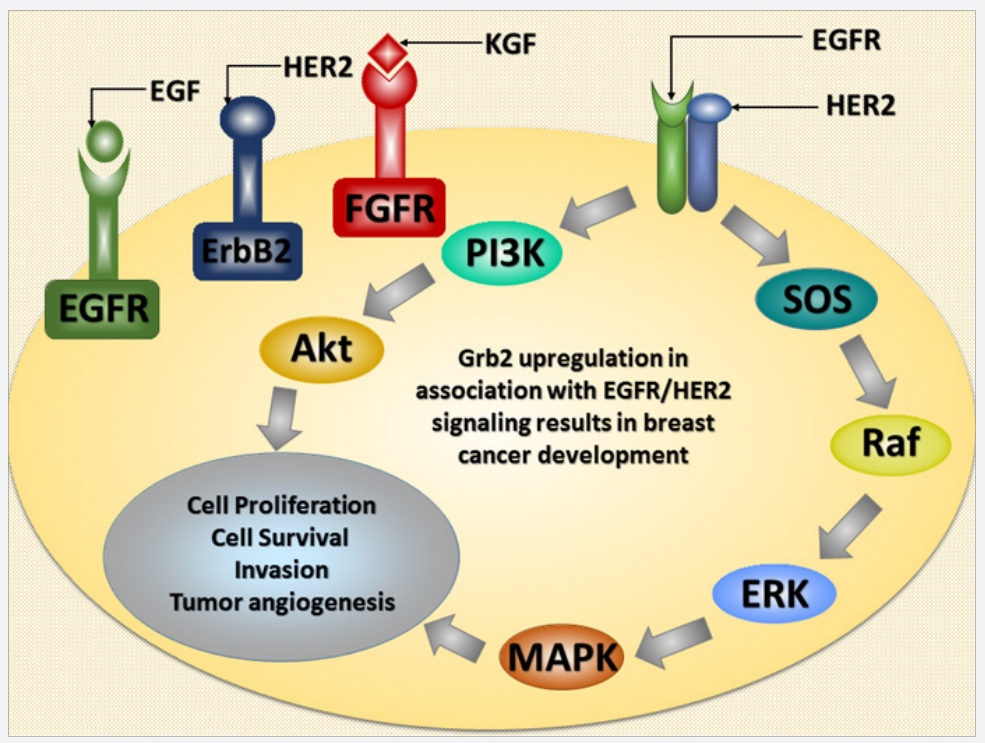

Figure 2: A model of aberrant Grb2 signaling in human breast cancer.

The Grb2 recruits a number of receptor tyrosine kinases such as EGFR, PDGFR etc. however, the Grb2 activation through some specific RTKs certify its involvement in breast cancer progression. The abnormal activation of Grb2 by Her2/erbB2 is related with the development of human breast cancers and mammary carcinoma. EGFR, Neu (Her2, erbB2) might utilize Grb2-mediated Akt or MAPK pathways to induce their oncogenic characteristics.

The signaling through a well-known transmembrane receptor tyrosine kinase, Neu (ErbB-2 or HER-2) has direct correlation in occurrence of 20 to $30 \%$ human mammary carcinomas (Figure 2). Neu has five autophosphorylation sites, two of which i.e. Y1144 (YB) and Y1227 (YD) are highly specific binding sites for adaptor proteins. Neu has capability to recruit the Grb2 directly through its Y1144 (YB) site while, indirectly by Shc to Y1227 (YD). Moreover, YB and YD sites are capable of efficiently inducing mammary tumors in female mice [22]. All-trans retinoic acid (ATRA) is capable to inhibit the growth of various cancer cells including breast cancer cells i.e. MCF-7 etc. However, Neu is thought to induce ATRA resistance in breast cancer cells. To test the same fact, MCF-7 cells were selected depending on having very low levels of HER-2/Neu. After 5 days of treatment with ATRA, a $63 \%$ inhibition in cell growth was noticed. Moreover, ATRA activity was tested on HER-2/Neu gene transfected MCF-7 cells and no concomitant ATRA induced anti-tumor activity was seen. Further investigation cleared that Grb2 and Akt proteins were responsible for the HER-2/Neu induced ATRA resistance, as Grb2 downregulation permitted ATRA to inhibit proliferation in breast cancer cells [23]. The transcription factor activating protein-1 (AP-1) is a DNA binding protein complex which is controlled by different growth factors and tumor promoters. The elevated AP-1 levels were observed in Grb2 mediated HER-2 overexpressing breast cancer cells. Moreover, the effects of Grb2 suppression were found in direct link to decrease the AP-1 binding activity in MCF-7/HER-2 cells [24]. Indeed, Grb2 is well known to link tyrosine kinase with Ras pathway, though earlier studies have indicated that Grb2 through SH3 domains have ability to bind various proteins like P228, P140, P55 and P28 other than SOS1, suggesting that Grb2 might has direct participation in other cellular functions. Nevertheless, higher amounts of Grb2-SOS1 complex in association with erb-B2 tyrosine kinase indicate its proximate relation in human breast cancer development [25].

Grb2 is originally present in the cell cytoplasm in abundant amounts while can also be found in the cell nucleus in minor extents. Verbeek et al. [26] has examined the Grb2 localization in normal versus tumor breast tissues. In normal breast tissue, $22 \%$ of Grb2 protein was detected in nuclei and $70 \%$ in cell cytoplasm. In tumor breast tissue, surprisingly $58 \%$ of $\mathrm{Grb} 2$ was found in nucleus while $37 \%$ in the cytosol. These findings revealed that Grb2 over expression in the human breast cancer resulted in its predominant localization in the cell nucleus [26]. Other than Grb2, several SH2 domain containing proteins such as c-Src, phospholipase C- $\gamma 1$ (PLC- $\gamma 1$ ), Grb7 and EMS1/ cortactin can also regulate EGFR and erb-B2 mediated signaling relevant to human breast cancers. These findings suggest that signaling proteins are of worthy since they can be used as prognostic indicators and might be helpful to target breast malignancies through a number of channels [27]. Grb2 and 


\section{Cancer Therapy \& Oncology International Journal}

PLC- $\gamma 1$ through their SH3 domains compete for the binding to C-terminal of fibroblast growth factor receptor 2 (FGFR2) and form concentration dependent complexes with receptor tyrosine kinase, this concentration dependent competition leads to the increased cell motility and invasion. Point mutations in the C-terminus of FGFR2 results in the formation of human breast cancer, gastric, bladder and stomach cancers [28]. Two members of ADP-ribosylation factor (ARF) family namely ARF1 and ARF6 act as activated downstream of EGFR signaling and regulate the proliferation, migration and invasion of breast cancer cells when regulated by the adaptor proteins p66Shc and Grb2 [29]. ARF1 is a small GTPase associated with Golgi, plays a critical role in migration and proliferation of breast cancer cells through Grb2-mediated PI3K signaling cascade [30]. While, ARF6 factor exhibits the migratory and proliferative activities of human breast cancer cells through ERK1, 2 pathway [31].

The protein-tyrosine phosphatase SHP-1 with two Src 2 domains is predominantly expressed in hematopoietic and epithelial cell lineages and plays a crucial role in both normal cellular events such as cell cycle, cell growth and as a regulator in pathological transformations [32]. Previously, SHP-1 mRNA expressions were investigated using 19 breast cancer cell lines and 72 primary breast cancers. Subsequent results showed 2-12 folds increase in SHP-1 mRNA expression in breast cancer cells as compared to normal breast epithelial cells in 58\% of samples. Likewise Grb2 mRNA expression was also found upregulated in breast cancer patients and surprisingly a positive correlation of SHP-1 mRNA expression in cancer specimens with that of Grb2 protein was observed [33]. Interestingly, SHP-1 and Grb2 mRNA were found in a significant relationship in which SHP-1 tyrosine residue (Tyr538) was located within the Grb2-SH2 binding motif PYXNX. Grb2 recruits a number of RTKs however, it is unable to engage PDGFR directly and utilizes the SHP-1 to mediate PDGFR signaling $[33,34]$. The exon skipped sequence in SHP-1 gene expression may lead to several hematopoietic abnormalities and systemic malignancies including ovarian cancer, prostate cancer and human breast cancer $[35,36]$.

Keratinocyte growth factor (KGF) is a member of FGFR family which is secreted by stromal cells and stimulates epithelial DNA synthesis, proliferation and migration in breast tissue. Previous reports have shown that KGF signal transduction increased the Grb2 expression by 2-3 folds and activate Erk1, 2 causing breast cancer cell motility [37]. KGF injected intravenously to female rats was found to cause dramatic proliferation of mammary epithelium in the mammary glands within 5 days [38]. Transforming growth factor- $\beta$ (TGF- $\beta$ ) is a cytokine that direct all stages of mammary gland development and also suppresses the mammary tumorigenesis. Phosphorylated Tyr284 of TGF- $\beta$ functions as SH2 domain binding site for Shc and Grb2, thereupon associating the oncogenic properties of TGF- $\beta$ with the docking Grb2 to T $\beta$ R-II [39]. Another cell surface receptor lemur tyrosine kinase 3 (LMTK3) binds directly to Grb2 and activates the Ras-mediated MAPK signaling. The overexpression of LMTK3 promoted the interaction between the Grb2 and SOS1 and contribute to breast cancer and metastasis [40].

\section{Grb2 antagonists}

The broad concern of Grb2 in various pathological complications makes it an impressive target for designing attractive anticancer schemes. Grb2 signaling can be targeted by blocking its connection to cell surface kinases via SH2 domain blockade or by targeting Grb2-SH3 domains and compromising it's interlink to downstream pathway.

As described earlier, tyrosine kinases such as EGFR, Neu (HER-2, erb-B2) through Grb2 protein act as the leading agents in breast cancer progression. Recent reports have shown that anti-HER2 directed therapies such as trastuzumab, pertuzumab and lapitinib have the ability to cure metastatic breast cancer by compromising the activity of Grb2 gene, however these agents might not be effective in all cases of breast cancers [41]. Another antidote named liposome-incorporated nuclease-resistant antisense oligodeoxynucelotide (L-Grb2) has high affinity for HER-2 mediated Grb2 signaling and can efficiently inhibit breast cancers. Further, downregulation of Grb2 expression was noticed to inhibit heregulin and erb-B2 stimulated breast cancer through Akt inactivation [42]. A similar report has indicated that the inactivation of EGFR regulated MAPK pathway through Grb2 down regulation induced the growth inhibition of breast cancer [43]. These findings suggest that different signaling pathways can be used by EGFR and erb-B2 to direct breast cancer growth.

p-malonylphenylanaline (Pmf), a novel phosphotyrosine (pTyr) mimetic holding high affinity for Grb2-SH2 domain has shown potent inhibition of Grb2-regulated erbB2-MAPK pathway in extracellular Grb2 binding assay. As cell penetration is a limitation for some chemotherapeutic agents with poor cell permeability, so the introduction of $\mathrm{N}$-oxalyl group to Pmf was evidenced to enhance its effectiveness intracellularly [44]. The Grb2 and SHP-1 broad relationship with different irregularities including human breast cancer has been discussed earlier. It is worth mentioning that stable gene expression of SHP-1 might be helpful to treat the cancer malignancies of hematopoietic cells [45].

Micro RNAs (miRNAs) are functional RNA molecules that control the expression of target genes and regulate the basic events of each cell. However, a minor alteration in the expression of miRNA leads to the development of different human cancers including lung cancer, colon cancer etc. [46]. Most recent studies have documented the involvement of downregulated miR411-5p expressions in progression of human breast cancer. Surprisingly, downstream Grb2 was found over expressed in breast cancer cells and that, Grb2 silencing by miR-411-5p suppressed the growth and metastasis in breast cancer cell via targeting Grb2-SOS-Ras pathway. Moreover, miR-411-5p inhibited the proliferation, invasion and progression of breast cancer in vitro and in vivo [47]. 


\section{Cancer Therapy \& Oncology International Journal}

The use of novel peptides as the Grb2 inhibitors in various cancer diseases can also act as an attractive approach in clinical sphere. Peptidimer-c activity against Grb2-SOS portion of HER-2 pathway was thoroughly investigated, subsequent results showed that potent peptide inhibited Grb2-mediated HER-2 overexpression in vitro, and induced anti-tumor effect via targeting Grb2-MAPK pathway in vivo. Moreover, the combination treatment of Peptidimer-c and docetaxel was correlated with the inhibition of both Akt and MAPK cascades [48]. Fmoc-Glu-Tyr-Aib-Asn-NH2, another novel peptide which has capability to target Grb2-SH2 domain in human breast cancer. The antagonistic activity of this peptide was investigated against MDA-MB-453 cells (cells with overexpressed erb-B2 expression driven through Grb2 signaling) and MCF-7 cells, concomitant data proved the anti-proliferative effects of peptide antagonist in breast cancer cells [49]. A fusion protein was constructed by joining PTD (an arginine rich peptide with ability to cross cell membrane) and Grb2-SH2 domain to target the EGFR and HER-2 via Grb2. The resultant PTD-Grb2-SH2 fusion protein was capable to enter the cytoplasm and nucleus and induced apoptosis in breast cancer cells and exhibited the sufficient toxicity to breast cancer cells in time and dose-dependent manner in vitro [50]. Free phosphonates are similar peptide-mimetic inhibitors which are also able to cross cell membrane barrier and inhibit cell motility in breast cancer cells by targeting Grb2-SH2 domain in vitro [51].

\section{Discussion}

Different adaptor proteins are well known for their key roles to regulate the diverse signaling pathways. Grb2 is one of a similar protein which has ability to connect cell membrane activated receptors to the downstream effectors. The mature Grb2 is a 217 amino acid molecule which was originally discovered for the regulation of normal cell functioning but wide range of documentation have proved the involvement of Grb2 in a number of cancer pathogenesis. Most recent studies have indicated the significant role of Grb2 in the T cell development and TH cell differentiation as well [3]. In fact, Grb2 negative regulatory role is more important as it can be advantageous to design potential therapeutic strategies. Grb2 can be tackle well either by targeting $\mathrm{SH} 2$ domain and compromising its connection to upstream tyrosine kinases or by blocking one of the two SH3 domains and thus inhibiting the downstream signaling.

This review provides a brief introduction of the Grb2 diverse functioning and its involvement in the development and progression of breast cancers and mammary carcinomas. Receptor tyrosine kinases such as EGFR, HER-2/erb-B2 act as important prognostic factors associated with breast cancer development when act through Grb2-SOS pathway [52]. Since, Grb2-SH2 domain plays an important role in this signaling therefore SH2 domain might act as an important therapeutic target for anticancer drug approaches. SHP-1 is another factor involved in the development of different cancers and also has a binding with Grb2-SH2 portion through SHP-1 tyrosine residue
(Tyr538). Likewise, abnormal signaling through several RTKs such as FGFR, PDGFR, LMTK3, T $\beta$ R-II etc. permits Grb2 to derive aberrant signaling result in various tumor conformations.

We have discussed the maximum possible ways to suppress the Grb2 signaling involved in human breast cancers and mammary carcinomas. As 20-30\% of all the human mammary carcinomas occurred due to the possible involvement of HER-2/erb-B2 kinases, so we have highlighted the potential chemotherapeutic agents which can inhibit Grb2-mediated HER2/erb-B2 pathway. Trastuzumab, pertuzumab, lapitinib, Pmf, peptidimer-c, PTD-Grb2-SH2 etc. have been identified as potent Grb2 antagonists which inhibit the H-ER-2/erb-B2 signaling in human breast cancer. Another molecule named miR-411-5p also has the ability to target the Grb2 in SOS-Ras signaling and inhibit the proliferation and invasion in human breast cancer cells. The insulin-like growth factor (IGF-IR) was also observed to control Grb2-MAPK induced breast cancer cell growth and tamoxifen was found to inhibit the growth of breast cancer cells overexpressing the IGF-IR [53].

\section{Conclusion}

In conclusion, we have compiled the maximum breast cancer malignancies caused by the possible involvement of the Grb2 adaptor. The Grb2 broad affiliation with a number of irregularities makes it a pivotal target to design therapeutic anticancer strategies. Previously indicated various Grb2 antagonists might be helpful to understand the approachable Grb2 targets and to build potential anticancer candidates to treat human breast cancer. Notwithstanding, further work is needed to pinpoint more distinctive docking sites of the Grb2 protein to target this adaptor through a different way.

\section{Acknowledgement}

Authors are grateful to Guy Temporal professional in Medical English proofreading, for his valuable help in proof $\neg$ reading and editing the manuscript.

\section{References}

1. Luo K (2017) Signaling Cross Talk between TGF- $\beta /$ Smad and Other Signaling Pathways. Cold Spring Harb Perspect Biol 9(1): a022137.

2. Ward AC, Smith L, de Koning JP, van Aesch Y, Touw IP (1999) Multiple signals mediate proliferation, differentiation, and survival from the granulocyte colony-stimulating factor receptor in myeloid 32D cells. J BiolChem 274(21): 14956-14962.

3. Radtke D, Lacher S M, Szumilas N, Sandrock L, Ackermann J, et al. (2016) Grb2 Is Important for T Cell Development, Th Cell Differentiation, and Induction of Experimental Autoimmune Encephalomyelitis. J Immunol 196(7): 2995-3005.

4. Yu GZ, Chen Y, Wang JJ (2009) Overexpression of Grb2/HER2 signaling in Chinese gastric cancer: their relationship with clinicopathological parameters and prognostic significance. J Cancer Res Clin Oncol 135(10): 1331-1339.

5. . Pendergast AM, Quilliam LA, Cripe LD, Bassing CH, Dai Z, et al. (1993) BCR-ABL-induced oncogenesis is mediated by direct interaction with the SH2 domain of the GRB-2 adaptor protein. Cell 75(1): 175-185. 


\section{Cancer Therapy \& Oncology International Journal}

6. Zhang Y, Li Z, Yang M, Wang D, Yu L, Guo C, et al. (2013) Identification of GRB2 and GAB1 co expression as an unfavorable prognostic factor for hepatocellular carcinoma by a combination of expression profile and network analysis. PLoS One: 8(12): e85170.

7. Watanabe T, Shinohara N, Moriya K, Sazawa A, Kobayashi Y, et al (2000) Significance of the Grb2 and son of sevenless (Sos) proteins in human bladder cancer cell lines. IUBMB Life 49(4): 317-320.

8. Timsah Z, Berrout J, Suraokar M, Behrens C, Song J, et al. (2015) Expression pattern of FGFR2, Grb2 and Plc 11 acts as a novel prognostic marker of recurrence recurrence-free survival in lung adenocarcinoma. Am J Cancer Res 5(10): 3135-3148.

9. Belov AA, Mohammadi M (2012) Grb2, a double-edged sword of receptor tyrosine kinase signaling. Sci Signal 5(249): pe49.

10. Pawson T (2007) Dynamic control of signaling by modular adaptor proteins. Curr Opin Cell Biol 19(2): 112-116.

11. Dharmawardana PG, Peruzzi B, Giubellino A, Burke TR, Bottaro DP (2006) Molecular targeting of growth factor receptor-bound 2 (Grb2) as an anti-cancer strategy. Anticancer Drugs 17: 13-20.

12. Christensen SM, Tu HL, Jun JE, Alvarez S, Triplet MG, et al. (2016) Oneway membrane trafficking of SOS in receptor-triggered Ras activation. Nat Struct Mol Biol 23(9): 838-846.

13. Chavan TS, Muratcioglu S, Marszalek R, Jang H, Keskin O, et al. (2016) Plasma membrane regulates Ras signaling networks. Cell Logist 5(4) e1136374.

14. Boguski MS, McCormick F (1993) Proteins regulating Ras and its relatives. Nature 366: 643-654.

15. Campbell SL, Khosravi-Far R, Rossman KL, Clark GJ, Der CJ (1998) Increasing complexity of Ras signaling. Oncogene 17: 1395-1413.

16. Bos JL (1989) ras Oncogenes in Human Cancer: A Review. Cancer Res 49(17): 4682-4689.

17. Ahmed Z, Timsah Z, Suen KM, Cook NP, Lee GR, et al. (2015) Grb2 monomer-dimer equilibrium determines normal versus oncogenic function. Nat Commun 6: 7354

18. Giubellino A, Burke TR, Bottaro DP (2008) Grb2 Signaling in Cell Motility and Cancer. Expert Opin Ther Targets 12(8): 1021-1033.

19. Torre LA, Bray F, Siegel RL, Ferlay J, Lortet-Tieulent J, et al.(2015) Global Cancer Statistics, 2012. CA Cancer J Clin 65(2): 87-108.

20. Kapoor S (2014) Grb2 and its influence on tumor growth and progression in systemic malignancies. Acta Oncol 53: 157-158.

21. Cheng AM, Saxton TM, Sakai R, Kulkarni S, Mbamalu G, et al. (1998) Mammalian Grb2 Regulates Multiple Steps in Embryonic Development and Malignant Transformation. Cell 95(6): 793-803.

22. Dankort D, Maslikowski B, Warner N, Kanno N, Kim H, et al. (2001) Grb2 and Shc Adapter Proteins Play Distinct Roles in Neu (ErbB-2)Induced Mammary Tumorigenesis Implications for Human Breast Cancer. Mol Cell Biol 21(5): 1540-1551.

23. Tari AM, Lim SJ, Hung MC, Esteva FJ, Lopez-Berestein G (2002) Her2/ neu induces all-trans retinoic acid (ATRA) resistance in breast cancer cells. Oncogene 21(34): 5224-5232.

24. Mendoza-Gamboa E, Siwak DR, Tari AM (2004) The HER2/Grb2/Akt pathway regulates the DNA binding activity of AP-1 in breast cancer cells. Oncol Rep 12(4): 903-908.

25. Sastry L, Cao T, King CR (1997) Multiple Grb2-protein complexes in human cancer cells. Int J Cancer 70(2): 208-213.

26. Verbeek BS, Adriaansen-Slot SS, Rijksen G, Vroom TM (1997) Grb2 overexpression in nuclei and cytoplasm of human breast cells: a histochemical and biochemical study of normal and neoplastic mammary tissue specimens. J Pathol 183(2): 195-203.

27. Kairouz R, Daly RJ (2000) Tyrosine kinase signalling in breast cancer Modulation of tyrosine kinase signalling in human breast cancer through altered expression of signalling intermediates. Breast Cancer Res 2(3): 197-202.

28. Timsah Z, Ahmed Z, Lin CC, Melo FA, Stagg LJ, et al. (2014) Competition between Grb2 and Plcy1 for FGFR2 regulates basal phospholipase activity and invasion. Nat StructMolBiol 21(2): 180-188.

29. Haines E, Saucier C, Claing A (2014) The Adaptor Proteins p66Shc and Grb2 Regulate the Activation of the GTPases ARF1 and ARF6 in Invasive Breast Cancer Cells. J Biol Chem 289(9): 5687-5703.

30. Boulay PL, Cotton M, Melançon P, Claing A (2008) ADP-ribosylation factor 1 controls the activation of the phosphatidylinositol 3-kinase pathway to regulate epidermal growth factor-dependent growth and migration of breast cancer cells. J Biol Chem 283(52): 36425-36434.

31. Hashimoto S, Onodera Y, Hashimoto A, Tanaka M, Hamaguchi M, et al. (2004) Requirement for Arf6 in breast cancer invasive activities. Proc Natl Acad Sci USA 101(17): 6647-6652

32. Tsui HW, Hasselblatt K, Martin A, Mok SC, Tsui FW (2002) Molecular mechanisms underlying SHP-1 gene expression. Eur J Biochem 269(12): 3057-3064.

33. Yip SS, Crew AJ, Gee JM, Hui R, Blamey RW, et al. (2000) Up-regulation of the protein tyrosine phosphatase SHP-1 in human breast cancer and correlation with GRB2 expression. Int J Cancer 88(3): 363-368.

34. Li W, Nishimura R, Kashishian A, Batzer AG, Kim WJ, et al. (1994) A New Function for a Phosphotyrosine Phosphatase: Linking GRB2-Sos to a Receptor Tyrosine Kinase. Mol Cell Biol: 14(1): 509-517.

35. Banville D, Stocco R, Shen SH (1995) Human protein tyrosine phosphatase 1C (PTPN6) gene structure: alternate promoter usage and exon skipping generate multiple transcripts. Genomics 27(1):16573.

36. Wu C, Sun M, Liu L, Zhou GW (2003) The function of the protein tyrosine phosphatase SHP-1 in cancer. Gene 306: 1-12.

37. Zang XP, Siwak DR, Nguyen TX, Tari AM, Pento JT (2004) KGF-induced motility of breast cancer cells is dependent on Grb2 and Erk1,2. ClinExp Metastasis 21(5): 437-443.

38. Ulich TR, Yi ES, Cardiff R, Yin S, Bikhazi N, et al. (1994) Keratinocyte growth factor is a growth factor for mammary epithelium in vivo. The mammary epithelium of lactating rats is resistant to the proliferative action of keratinocyte growth factor. Am J Pathol 144(5): 862-868.

39. Galliher-Beckley AJ, Schiemann WP (2008) Grb2 Binding to Tyr284 in T $\beta R$-II is Essential for Mammary Tumor Growth and Metastasis Stimulated by TGF- $\beta$. Carcinogenesis 29(2): 244-251.

40. Xu Y, Zhang H, Lit LC, Grothey A, Athanasiadou M, et al. (2014) The kinase LMTK3 promotes invasion in breast cancer through GRB2 mediated induction of integrin $\beta_{1}$. Sci Signal 7(330): ra58.

41. Park YH, Shin HT, Jung HH, Choi YL, Ahn T, et al. (2015) Role of HER2 mutations in refractory metastatic breast cancers: targeted sequencing results in patients with refractory breast cancer. Oncotarget 6(31): 32027-32038.

42. Lim SJ, Lopez-Berestein G, Hung MC, Lupu R, Tari AM (2000) Grb2 downregulation leads to Akt inactivation in heregulin-stimulated and ErbB2-overexpressing breast cancer cells. Oncogene 19(54): 62716276.

43. Tari AM, Hung MC, Li K, Lopez-Berestein G (1999) Growth inhibition of breast cancer cells by Grb2 downregulation is correlated with 


\section{Cancer Therapy \& Oncology International Journal}

inactivation of mitogen-activated protein kinase in EGFR, but not in ErbB2 cells. Oncogene 18(6): 1325-1332.

44. Gao Y, Luo J, Yao ZJ, Guo R, Zou H, et al. (2000) Inhibition of Grb2 SH2 Domain Binding by Non-Phosphate-Containing Ligands. 2. 4-(2-Malonyl) phenylalanine as a Potent Phosphotyrosyl Mimetic. J Med Chem 43(5): 911-920.

45. Zapata PD, Ropero RM, Valencia AM, Buscail L, López JI, et al. (2002) Autocrine Regulation of Human Prostate Carcinoma Cell Proliferation by Somatostatin through the Modulation of the SH2 Domain Containing Protein Tyrosine Phosphatase (SHP)-1. J Clin Endocrinol Metab 87(2): 915-926.

46. Shenouda SK, Alahari SK (2009) MicroRNA function in cancer: oncogene or a tumor suppressor? Cancer Metastasis Rev 28(3-4): 369 378

47. Zhang Y, Xu G, Liu G, Ye Y, Zhang C, et al. (2016) miR-411-5p inhibits proliferation and metastasis of breast cancer cell via targeting GRB2. Biochem Biophys Res Commun 476(4): 607-613.

48. Gril B, Vidal M, Assayag F, Poupon MF, Liu WQ, et al. (2007) Grb2-SH3 ligand inhibits the growth of HER21 cancer cells and has antitumor effects in human cancer xenografts alone and in combination with docetaxel. Int J Cancer 121(2):407-415.

49. Chen CH, Chen MK, Jeng KC, Lung FD (2010) Effects of Peptidic Antagonists of Grb2-SH2 on Human Breast Cancer Cells. Protein Pept Lett 17(1): 44-53.

50. Yin J, Cai Z, Zhang L, Zhang J, He X, et al. (2013) A recombined fusion protein PTD-Grb2-SH2 inhibits the proliferation of breast cancer cells in vitro. Int J Oncol 42(3): 1061-1069.

51. Morlacchi P, Robertson FM, Klostergaard J, McMurray JS (2014) Targeting SH2 domains in breast cancer. Future Med Chem 6(17): 1909-1926.

52. Timms JF, White SL, O'Hare MJ, Waterfield MD (2002) Effects of ErbB2 overexpression on mitogenicsignalling and cell cycle progression in human breast luminal epithelial cells. Oncogene 21(43): 6573-6586.

53. Guvakova MA, Surmacz E (1997) Tamoxifen Interferes with the Insulin-like Growth Factor I Receptor (IGF-IR) Signaling Pathway in Breast Cancer Cells. Cancer Res 57(13): 2606-2610.

Your next submission with Juniper Publishers will reach you the below assets

- Quality Editorial service

- Swift Peer Review

- Reprints availability

- E-prints Service

- Manuscript Podcast for convenient understanding

- Global attainment for your research

- Manuscript accessibility in different formats

( Pdf, E-pub, Full Text, Audio)

- Unceasing customer service

Track the below URL for one-step submission https://juniperpublishers.com/online-submission.php 